SUBJECT AREAS: MATERIALS SCIENCE NANOSCIENCE AND TECHNOLOGY

PHOTOCATALYSIS

NANOPHOTONICS AND PLASMONICS

Received 13 March 2013

Accepted

12 June 2013

Published 5 July 2013

Correspondence and requests for materials should be addressed to

P.K.S. (stsspk@mail. sysu.edu.cn) or S.P.J. (s.jiang@curtin.edu.

\section{Large-scale and Rapid Synthesis of Disk-Shaped and Nano-Sized Graphene}

\author{
Chunyong $\mathrm{He}^{1}$, San Ping Jiang ${ }^{2}$ \& Pei Kang Shen ${ }^{1}$
}

\begin{abstract}
'State Key Laboratory of Optoelectronic Materials and Technologies, and Guangdong Province Key Laboratory of Low-carbon Chemistry \& Energy Conservation, School of Physics and Engineering, Sun Yat-sen University, Guangzhou, 510275, PR China, ${ }^{2}$ Fuels and Energy Technology Institute \& Department of Chemical Engineering, Curtin University, Perth, WA6102, Australia.
\end{abstract}

We synthesized disk-shaped and nano-sized graphene (DSNG) though a novel ion-exchange methodology. This new methodology is achieved by constructing metal ion/ion-exchange resin framework. The morphology and size of the graphene can be modulated by changing the mass ratio of the carbon-containing resin to the cobalt-containing precursor. This is the first time to show that the DSNG formed on the granular transition metal substrate. The DSNG gives a high intensity of photoluminescence at near-UV wavelength of $311 \mathrm{~nm}$ which may provide a new type of fluorescence for applications in laser devices, ultraviolet detector UV-shielding agent and energy technology. The emission intensity of the DSNG is thirty times higher than that of the commercial large graphene. Our approach for graphene growth is conveniently controllable, easy to scale-up and the DSNG shows superior luminescent properties as compared to conventional large graphene.

$\mathrm{t}$ is well-known from many theoretical and experimental evidences that nanographene with specifically morphologies have excellent properties for broad application prospects ${ }^{1-4}$. The applications of graphene have been widely studied ${ }^{4-6}$. The amazing properties of graphene make it a likely candidate to spur on the next revolution in electronics ${ }^{7}$. However, large-area graphene flakes are not semiconductor because there is no energy gap. But its energy gap can be opened when graphene is shaped up into quasi-one-dimensional structures with narrow widths $(<\sim 30 \mathrm{~nm})$, and similar to CNTs, the energy gap of graphene is tunable from 0 to $0.5 \mathrm{eV}$, depending on the width and crystallographic orientation of the graphene nanoribbon $(\mathrm{GNR})^{2,8}$. Graphene also touted as the basis for next generation spintronics devices ${ }^{9,10}$. Arbitrarily shaped finite graphene nanoflakes (GNFs) can lead to large net spin or antiferromagnetic coupling between groups of electron spins. And the spin of GNFs depends on its shape due to topological frustration of the $\partial$-bonds ${ }^{11-13}$. Large area $(>100 \mathrm{~nm})$ graphene behaves as conventional single-electron transistors, exhibiting periodic Coulomb blockade peaks. When the size of graphene narrows down to $100 \mathrm{~nm}$, even sub-10 nm, which refer as graphene quantum dots (GQDs), the peaks become strongly nonperiodic, indicating a major contribution of quantum confinement ${ }^{14}$. The GQDs with sub-10 nm in width remain conductive and reveal a confinement gap of up to $0.8 \mathrm{eV}$, demonstrating the possibility of molecular-scale electronics based on graphene ${ }^{15,16}$. Apart from potential applications for the topdown approach to molecular-scale electronics, GQDs also have other unique properties and potential applications in many fields, such as photovoltaic devices ${ }^{4}$, biological label ${ }^{17}$, light emitting diodes ${ }^{18}$, and sensor ${ }^{19}$.

Although various effective techniques, such as mechanical exfoliation ${ }^{1}$, oxidation of graphite ${ }^{20}$, liquid-phase exfoliation ${ }^{21}$, epitaxial growth ${ }^{22}$ and thermal decomposition of $\mathrm{SiC}^{23}$ have been developed to produce single-layer or few-layers graphene, the controlled method of preparing nanographene still counts for the top issue ${ }^{24}$. Han et $a .^{2}$ used lithography etching technology lithographically patterned graphene nanoribbon (GNR) structures. Tapaszo et al. ${ }^{8}$ successfully obtained well-defined nanoribbons with widths down to $2.5 \mathrm{~nm}$ by applying a constant bias potential (significantly higher than the one used for imaging) and simultaneously moving the STM tip with constant velocity. Campos et al..$^{25}$ demonstrated anisotropic etching of the single-layer graphene by thermally activated nickel nanoparticles and obtain sub-10-nm nanoribbons. Dai et al. ${ }^{26}$ developed a route in which multi-walled carbon nanotubes were partially embedded in a PMMA film and then etched the exposed tube walls by argon plasma. The resulting nanoribbons have smooth edges and very narrow width (10-20 nm). Pan et $a l .{ }^{27}$ have developed a hydrothermal route for cutting preoxidized graphene sheets into GQDs (9.6 $\mathrm{nm}$ in average diameter) with blue emissions. Lu et al. ${ }^{15}$ demonstrated the formation of regularly sized graphene quantum dots on $\mathrm{Ru}(0001)$ substrate by using $\mathrm{C}_{60}$, which acts as a unique precursor compared to $\mathrm{C}_{2} \mathrm{H}_{4}$. Zhuo et al. ${ }^{28}$ presented a new facile ultrasonic method to prepare GQDs, which exhibited bright PL in a water solution. 


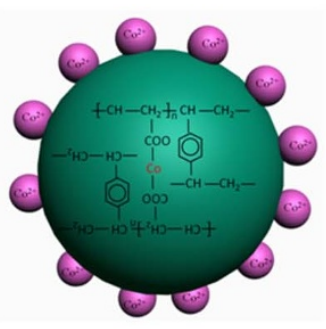

D113 located cobalt ions

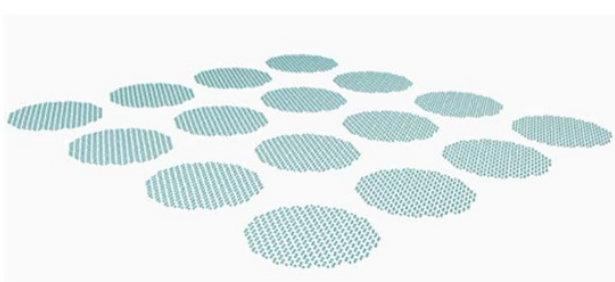

DSNG

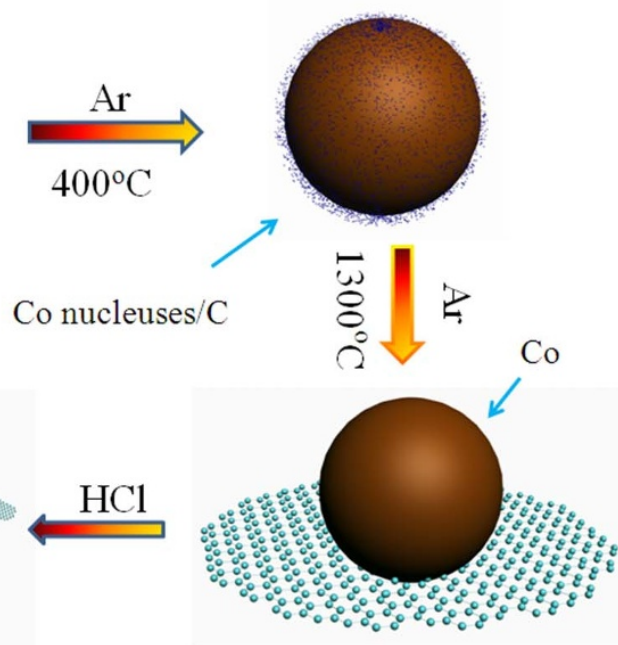

DSNG

Figure $1 \mid$ Schematic view of the approach for disk-shaped and nano-sized graphene (DSNG) growth.

To our best knowledge, the disk-shaped and nano-sized graphene (DSNG) has rarely been reported. The arduousness of obtaining DSNG with well-defined shape is the main reason of the poverty in study. Although some techniques, such as chemically derived techniques $^{29}$, chemical vapor deposition on template ${ }^{30}$ and lithography etching ${ }^{31}$ have been reported, however, effective method to produce DSNG with well-defined shape remains elusive. The diskshaped and nano-sized structure makes the DSNG possess much higher edge density and fringe defects than that of normal graphene sheets, which will contribute to better edge reactivity and defectsmediated properties ${ }^{31,32}$. The structural defects may give rise to localized electronic states and lead to the magnetic state in DSNG ${ }^{32-34}$. These can offer key advantages in realizing various electron applications via edge chemical functionalization, such as doping.

Recently, graphene based nanomaterial ${ }^{35}$ are attracting more and more attentions as luminescent nanomaterials due to their unique properties, such as low toxicity, high luminescent, robust chemical inertness and environmentally-friendly.

In this paper, we directly synthesized DSNG though a whole new methodology. The new methodology is achieved by constructing metal ion-ion exchange resin framework. The high quality of DSNG was confirmed by atomic force microscopy (AFM), scanning electron microscopy (SEM), Raman, X-ray Diffraction (XRD) and Xray photoelectron spectroscopy (XPS). Our approach for DSNG growth is actually utmost simple, conveniently controllable and easily scale-up. The synthetic process of the DSNG is shown in Figure 1.

\section{Results}

The AFM was used to characterize the material deposited on newdelaminated mica substrate at ambient conditions. It is observed that the material is disk-shaped with the diameter around $200 \mathrm{~nm}$ (Figure 2a). The DSNG gave the appearance of the uniform disks. To obtain the morphology and structure of the DSNG, we measured the thickness of the sample by AFM. Figure 2b shows the AFM result that the nano-sized graphene were disk-shaped with the height of $0.74 \mathrm{~nm}$. Figure $2 \mathrm{c}$ displays the scanning electron microscope (SEM) of the as-grown DSNG which are well dispersed. As shown in Figure 2d, a perfect disk-like shape can be clearly seen in the SEM micrograph with the size around $200 \mathrm{~nm}$.

The D113 resin exchanged with cobalt ions was heated in tube furnace at $400^{\circ} \mathrm{C}$ under Ar atmosphere for $2 \mathrm{~h}$, resulting in amorphous powder which was confirmed by the XRD measurements (Figure S3, pattern a). This step is essential in the synthetic process of DSNG, otherwise, no inerratic DSNG is observed (Figure S2). When the sample was further heat treated at $1300^{\circ} \mathrm{C}$, the polycrystalline cobalt particles and graphitic carbon formed (Figure S3, pattern b). The Co was removed by $\mathrm{HCl}$ treatment for $12 \mathrm{~h}$ (Figure S3, pattern c).

The Raman spectra of the HOPG and DSNG in the range of 1000$3000 \mathrm{~cm}^{-1}$ are shown in Figure 3a. The band observed at about $1577 \mathrm{~cm}^{-1}$ is $\mathrm{G}$ band, which corresponds to a splitting of the $E_{2 \mathrm{~g}}$ stretching mode of graphite and reflects the structural intensity of the $\mathrm{sp}^{2}$-hybridized carbon atom. The $1350 \mathrm{~cm}^{-1}$ band (D band), assigned to zone center phonons of $\mathrm{K}$-point phonons of $\mathrm{A}_{1 \mathrm{~g}}$ symmetry, characterizes the disordered graphite planes and the defects incorporated into pentagon and heptagon graphitic structures. The minor D band (Figure 3a) in DSNG mainly due to the huge amounts
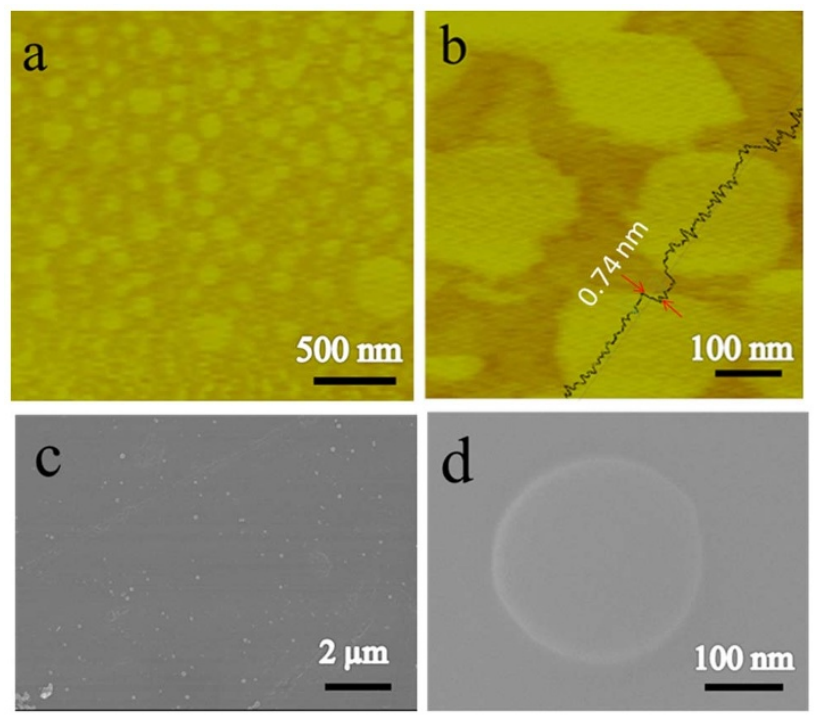

Figure $2 \mid$ Microscopic images of DSNG. (a). The tapping mode AFM image of the disk-shaped nano-sized graphene (DSNG) prepared at mass ratio of $\mathrm{CoCl}_{2} \cdot 6 \mathrm{H}_{2} \mathrm{O}$ to $\mathrm{D} 113$ resin $\left(\mathrm{R}_{\mathrm{Co} / \mathrm{R}}\right)$ of 0.1 , (b). a close-up of DSNG, (c). SEM micrograph of DSNG and (d). high resolution SEM micrograph of a DSNG. 

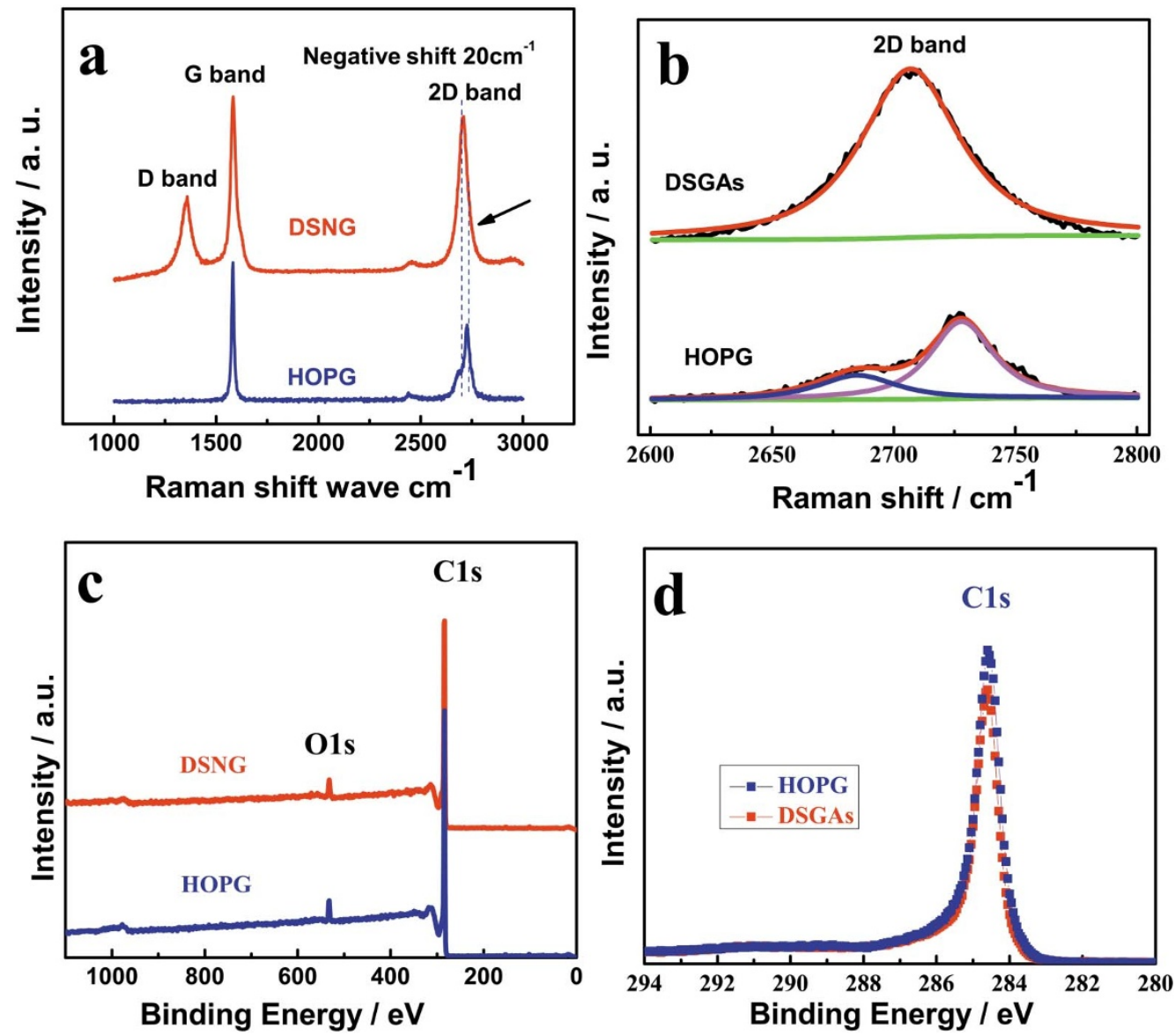

Figure 3 Raman and XPS spectra of DSNG. (a). Comparison of Raman spectra of DSNG (red line) and highly oriented pyrolytic graphite (HOPG) (blue line) at $514 \mathrm{~nm}$, (b). the 2D band in the Raman spectrum obtained from DSNG is formed by one Lorentzian peak, and the HOPG is formed by a two-peak line shape, (c). comparison of XPS spectra of DSNG (red line) and HOPG (blue line) and (d). high-resolution C1s XPS spectra of DSNG (red line) and HOPG (blue line).

of edges ${ }^{36}$. The result shows that a significant change in shape and intensity of the 2D band of DSNG compared to HOPG (Figure 3b). The 2D band in the Raman spectrum obtained from HOPG is formed by a two-peak line shape, consisting of two components $2 \mathrm{D}_{1}$ and $2 \mathrm{D}_{2}{ }^{37}$. The $2 \mathrm{D}$ band in the Raman spectrum of DSNG is a single and sharp peak, formed by one Lorentzian peak ${ }^{38}$. Notably, the intensities of the $2 \mathrm{D}$ band and $\mathrm{G}$ band for DSNG are comparable. The $2 \mathrm{D}$ position of DSNG is $20 \mathrm{~cm}^{-1}$ shift to lower frequency than that of the HOPG.

$\mathrm{X}$-ray photoelectron spectroscopy (XPS) is a powerful spectroscopic technique that measures the elemental composition, empirical formula, chemical state and electronic state of the elements existed in a material. XPS was used to study the bonding state of carbon atom in the DSNG. The full scan spectra of the DSNG are shown in Figure 3c. The peaks at binding energy of $284.6 \mathrm{eV}$ and $533.15 \mathrm{eV}$ are ascribed to $\mathrm{C} 1 \mathrm{~s}$ and O1s. The O1s is ascribed to the adsorbed molecular oxygen on the surface of the DSNG and HOPG. The hybridization of carbons influences the properties of a carbon nanomaterial and has implications in its structure. XPS can be used to determine the hybridization of the carbons on the surface of a material, such as graphite and nanodiamond. Graphite is a carbon material consisting of $\mathrm{sp}^{2}$ carbons. Theoretically the XPS of the pure graphite will show a single $\mathrm{C} 1 \mathrm{~s}$ peak, with a binding energy characteristic of $\mathrm{sp}^{2}$ carbon. Figure 3d shows the detailed C1s XPS spectrum of DSNG (red line) and HOPG (blue line). The main peak at $284.6 \mathrm{eV}$ corresponds to the graphite-like $\mathrm{sp}^{2}$ bonded carbon ${ }^{39}$. The $\mathrm{C} 1 \mathrm{~s}$ spectrum of DSNG coincides with HOPG, indicating most of the carbon atoms in DSNG are arranged in a conjugated honeycomb lattice ${ }^{39}$.
Figure 4 demonstrates that the morphology of the samples is a function of mass ratio of $\mathrm{CoCl}_{2} \cdot 6 \mathrm{H}_{2} \mathrm{O}$ to $\mathrm{D} 113$ resin $\left(\mathrm{R}_{\mathrm{Co} / \mathrm{R}}\right)$. It was found that the samples display different geography and size when the $\mathrm{R}_{\mathrm{Co} / \mathrm{R}}$ changes. In the case of the $\mathrm{R}_{\mathrm{Co} / \mathrm{R}}=0.2$ and 0.5 , there are two kinds of DSNG coexisted with different dimensions of the samples (Figure $4 \mathrm{a}$ and $4 \mathrm{~b}$ ). One is bigger and another one is very small. When the $\mathrm{R}_{\mathrm{Co} / \mathrm{R}}=1$, the geography of the graphene is inclined to become random (Figure 4c). The AFM height analysis illustrates that the heights of all the samples are between $0.45 \mathrm{~nm}$ to $0.71 \mathrm{~nm}$ (see

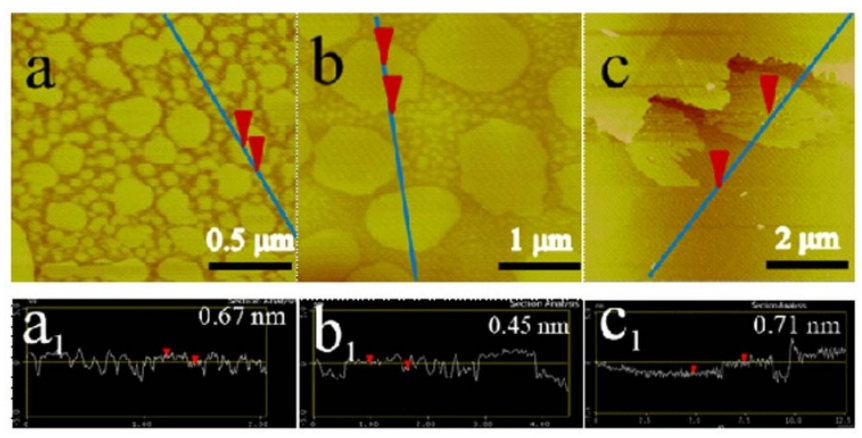

Figure $4 \mid$ AFM images of the gaphene samples. The tapping mode AFM images of the graphene samples deposited on new-delaminated mica at ambient condition as a function of mass ratio of $\mathrm{CoCl}_{2} \cdot 6 \mathrm{H}_{2} \mathrm{O}$ to D113 resin: (a). $\mathrm{R}_{\mathrm{Co} / \mathrm{R}}=0.2,(\mathrm{~b}) \cdot \mathrm{R}_{\mathrm{Co} / \mathrm{R}}=0.5$ and (c). $\mathrm{R}_{\mathrm{Co} / \mathrm{R}}=1$. The $\mathrm{a}_{1}-\mathrm{c}_{1}$ are the height profiles along the blue lines in $(\mathrm{a}-\mathrm{c})$. respectively. 


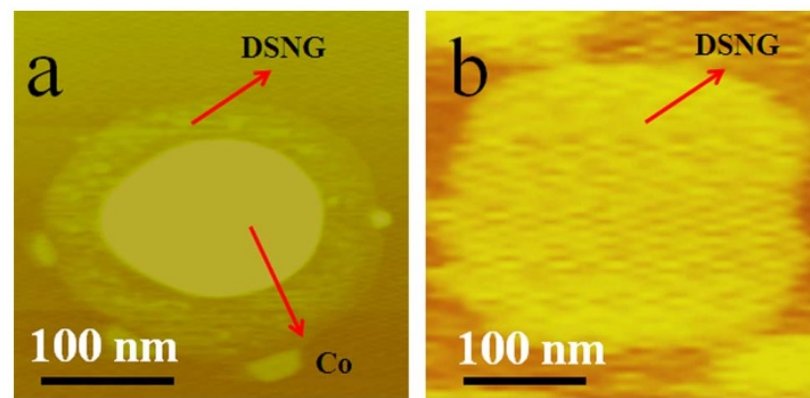

Figure $5 \mid$ AFM images of DSNG. (a). The tapping mode AFM image of the Co/graphene before acid treatment $\left(\mathrm{R}_{\mathrm{Co} / \mathrm{R}}=0.1\right)$ and $(\mathrm{b})$. the tapping mode AFM image of DSNG after acid treatment $\left(\mathrm{R}_{\mathrm{Co} / \mathrm{R}}=0.1\right)$.

Figure $4 a_{1}-c_{1}$ ). The Raman spectra (Figure S4) demonstrate that all the samples possess the characteristics of graphene. The XPS spectra (Figure S5) of the samples show that the carbon atoms in the samples are $\mathrm{sp}^{2}$ hybridized carbon in the regular hexagon plane, respectively.

The DSNG is formed by the catalysis of cobalt during the heat treatment which is confirmed by the AFM measurements before and after acid treatment. Figure 5 shows the typical results on a sample prepared in the case of the $\mathrm{R}_{\mathrm{Co} / \mathrm{R}}$ value of 0.1 . Figure $5 \mathrm{a}$ presents a disk there a Co particle located on the surface of graphene. The peripheral graphene disk is lighter yellow and the Co particle gives darker color, respectively. A flat disk formed after the removal of the Co particle as shown in Figure 5b. The AFM images of the Co on graphene samples with $\mathrm{R}_{\mathrm{Co} / \mathrm{R}}=0.1,0.2,0.5$ and 1 are shown in Figure S6 and S7.

Highly efficient luminescent materials hold the key for applications in optoelectronic devices, biological labeling and sensing ${ }^{40-42}$. Carbon-based nano-materials have been attracting the leading attention as they show high efficiency, stable emissions, chemical inertia, nontoxicity in the physiological condition and no environmental pollution ${ }^{43}$. With this in mind, we recorded the photoluminescent excitation (PLE) spectrum and PL emission spectrum of DSNG, and the large-area graphene was also tested. A PL emission with high intensity observed from DSNG shows a near-UV wavelength at $311 \mathrm{~nm}(3.98 \mathrm{eV})$ under an excitation wavelength of $270 \mathrm{~nm}$ $(4.62 \mathrm{eV})$ (Figure 6b). The emission intensity of DSNG is thirty times as high as that of large-area graphene. The enhanced emission indicates that there are more emission active sites. Thus, we expect that the nanostructured DSNG of $\mathrm{sp}^{2}$ configuration of similar size is likely to be responsible for the enhanced near-UV PL. The PLE spectrum for DSNG is shown in Figure 6a. Excitonic features are readily observable between excitation wavelengths of 240 and $295 \mathrm{~nm}$, which represent the absorption energies corresponding to emission of near- $\mathrm{UV}^{44}$. Figure S8 shows the Photograph of suspension of DSNG $\left(1 \mathrm{mg} \mathrm{ml}^{-1}\right)$ in $\mathrm{H}_{2} \mathrm{O}, \mathrm{N}$-methyl-2-pyrrolidone (NMP), N,N-Dimethylformamide (DMF) and 1,2-Dichloroethane (EDC).

The PL performance of functionalized graphene is related with the size, shape, defects, and preparation method. Table S1 displays different examples of the photoluminescence from functionalized graphene. Figure $6 c$ compares the PL for the graphene samples derived from different $\mathrm{R}_{\mathrm{Co} / \mathrm{R}}$. All the peaks are in the same near-UV. The emission intensity increases with the decreasing of $\mathrm{R}_{\mathrm{Co} / \mathrm{R}}$ (Figure $6 \mathrm{~d}$ ). The origin of photoluminescence (PL) in these carbon-based nanomaterials is tentatively proposed to be from isolated polyaromatic structures or passivated surface defects ${ }^{43}$. These results show that the state of the active emission point of the samples are the same. The increase in the emission intensity is mainly due to the increase in the fringe defects which result from the decrease of graphene sheet size. Figure S9 shows the relationship between the normalized PL intensity and the graphene size. The emission intensity decreases with the increase of the graphene size.
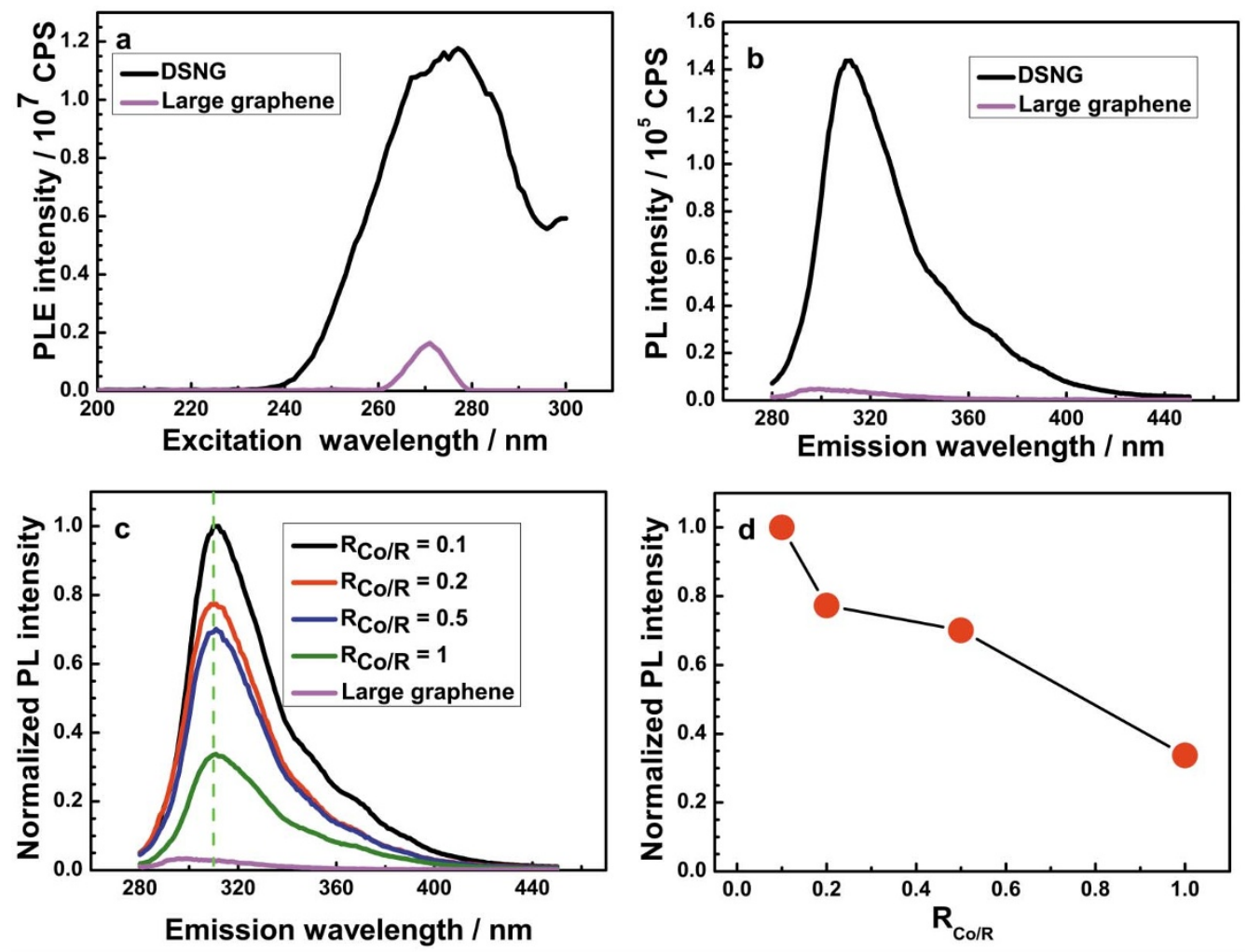

Figure $6 \mid$ PL spectra of DSNG. (a). PLE spectra of the DSNG $\left(\mathrm{R}_{\mathrm{Co} / \mathrm{R}}=0.1\right)$ and large-area graphene with the detection wavelength of $311 \mathrm{~nm}$ and $(\mathrm{b})$. PL spectra of the DSNG $\left(R_{\mathrm{Co} / \mathrm{R}}=0.1\right)$ and large-area graphene excited at $270 \mathrm{~nm},(\mathrm{c})$. PL spectra of the nanographene samples with different $\mathrm{R}_{\mathrm{Co} / \mathrm{R}}$ and $(\mathrm{d})$. the relationship between the normalized PL intensity and the $\mathrm{R}_{\mathrm{Co} / \mathrm{R}}$. 


\section{Discussion}

The synthesis of large area, homogenous graphene on cobalt by chemical vapor deposition CVD method has been reported ${ }^{45-48}$. The synthetic mechanism of cobalt catalyzed graphene by CVD is shown as follows. A solid solution of cobalt and carbon is formed when the mixture of $\mathrm{H}_{2}$ and amorphous gaseous carbon (a-C) source is introduced to the hot cobalt film. In the process of cooling down, the high energy carbon atoms in cobalt metal overcome the energy barrier and segregate from the interior of the cobalt film to the surface to form graphene $e^{45,47}$. The graphene grown by CVD on cobalt substrate is extensive,low-defect and homogeneous. Our approach for DSNG growth is schematically illustrated in Figure 1. The D113 resin exchanged with metal ions were carbonized in tubular furnace at $400^{\circ} \mathrm{C}$ under $\mathrm{Ar}$ atmosphere for $2 \mathrm{~h}$. In this process, the cobalt atoms, encapsulated in the exchange sites of the D113 resin, bring together into nucleuses. Simultaneously, the D113 resin began decomposition. After going through a carbonization process, the a$\mathrm{C}$, derived from the carbonization of the D113 resin, adsorb on the surface of the cobalt nucleuses. The compound is still amorphous solids (Figure S3 pattern a). Next, the compound was then heated to $1300^{\circ} \mathrm{C}$ for $1 \mathrm{~h}$ under $\mathrm{Ar}$ atmosphere condition. At this stage, the cobalt nucleuses grow and become polycrystalline cobalt particles with the increase of the temperature (Figure S3, pattern b). The sample was then cooled down to room temperature. In the process of cooling, the carbon atoms diffuse out of the cobalt to the surface, forming the nanographene. These infant nanographenes are expanding outward homogeneously at all orientations, forming a series of inerratic DSNG (Figure 5a).

In conclusion, we reported a novel ion-exchange method to synthesize the DSNG with superior luminescent properties. This is the first example to show that the DSNG formed under the catalysis of the granular cobalt. The morphology and size of the graphene can be modulated by changing the mass ratio of the cobalt-containing precursor to the carbon-containing resin. The DSNG synthesized in this work showed high efficiency in nearUV luminescence which may provide a new type of fluorescence for applications in laser devices, ultraviolet detector UV-shielding agent and energy technology. They may expand the application of graphene-based materials to other fields. Our approach for graphene growth is conveniently controllable, easy to scale-up and readily for applications.

\section{Methods}

Preparation of disk-shaped and nano-sized graphene. In a typical preparation, $2.0 \mathrm{~g} \mathrm{CoCl}_{2} .6 \mathrm{H}_{2} \mathrm{O}$ was dissolved in $200 \mathrm{ml}$ deionized water and $20 \mathrm{~g} \mathrm{D} 113$ resin was put into the solution (the mass ratio of $\mathrm{CoCl}_{2} \cdot 6 \mathrm{H}_{2} \mathrm{O}$ to $\mathrm{D} 113$ resin: $\mathrm{R}_{\mathrm{Co} / \mathrm{R}}=0.1$ ). After magnetic stirring for $2 \mathrm{~h}$, the D113 resin loaded with metal ions were rinsed with deionized water for several times and dried in oven at $80^{\circ} \mathrm{C}$. Figure $\mathrm{S} 1$ shows the typical profile of the ion exchange process of $\mathrm{D} 113$ between $\mathrm{Co}_{2}{ }^{+}$and $\mathrm{H}^{+}$. After carbonized in a tube furnace at $400^{\circ} \mathrm{C}$ under Ar atmosphere condition for $2 \mathrm{~h}$, the samples were further heat treated in tube furnace at $1300^{\circ} \mathrm{C}$ for $1 \mathrm{~h}$. The product was post-treated in $3.0 \mathrm{~mol} \mathrm{~L}^{-1} \mathrm{HCl}$ solution for more than $12 \mathrm{~h}$ with magnetic stirring to remove cobalt on the surface of the graphene.

The other graphene samples with different morphologies are synthesized by the same steps as mentioned above. In these preparations, $4.0 \mathrm{~g}, 8.0 \mathrm{~g}, 20 \mathrm{~g} \mathrm{CoCl}_{2} \cdot 6 \mathrm{H}_{2} \mathrm{O}$ was dissolved in $200 \mathrm{ml}$ deionized water and $20 \mathrm{~g} \mathrm{D113}$ resin was put into the solution $\left(\mathrm{R}_{\mathrm{Co} / \mathrm{R}}=0.2,0.5\right.$ and 1$)$, respectively. After treated by the same steps mentioned above. The graphene samples with different morphologies were obtained.

More details on the experimental section are presented in the Supplementary Information.

Characterizations. The X-ray diffraction (XRD) analysis was performed on a D/ Max-III (Rigaku Co., Japan) using $\mathrm{Cu} \mathrm{K} \alpha$ radiation, and operating at $40 \mathrm{kV}$ and $30 \mathrm{~mA}$. The $2 \theta$ angular regions between $10^{\circ}$ and $110^{\circ}$ were explored at a scan rate of $5^{\circ} \mathrm{min}^{-1}$. The Raman spectroscopic measurements were carried out on a Raman spectrometer (Renishaw Corp., UK) using a He/Ne laser with a wave length of $514.5 \mathrm{~nm}$. The X-ray photoelectron spectroscopy (XPS) measurements were carried out on a XPS apparatus (ESCALAB 250, Thermo-VG Scientific Ltd.). A field emission scanning electron microscope (FESEM,JSM-6330F, JEOL Ltd.) and multimode scanning probe microscope (SPM, Nanoscop IIIa, Digital Instrument Ltd.). PL spectra were measured at room temperature in a FLS920P system (EDINBURGH INSTRUMENTS Ltd.) with a $450 \mathrm{~mW}$ xenon arc lamp. All the samples were diluted with DI water to avoid reabsorption; the samples were placed in the cuvettes inside the integrating sphere.

1. Son, Y.-W., Cohen, M. L. \& Louie, S. G. Half-metallic graphene nanoribbons. Nature 444, 347-349 (2006).

2. Han, M., Özyilmaz, B., Zhang, Y. \& Kim, P. Energy band-gap engineering of graphene nanoribbons. Phys. Rev. Lett. 98, 206805 (2007).

3. Rao, C. N. R., Sood, A. K., Subrahmanyam, K. S. \& Govindaraj, A. Graphene: the new two-dimensional nanomaterial. Angew. Chem. Int. Ed. 48, 7752-7777 (2009).

4. Zhang, Z., Zhang, J., Chen, N. \& Qu, L. Graphene quantum dots: an emerging material for energy-related applications and beyond. Energy Environ. Sci. 5, 8869-8890 (2012).

5. Han, T. H. et al. Peptide/Graphene hybrid assembly into core/shell nanowires. Adv. Mater. 22, 2060-2064 (2010).

6. Hwang, J. O. et al. Workfunction-tunable, N-doped reduced graphene transparent electrodes for high-performance polymer light-emitting diodes. ACS Nano 6, 159-167 (2011).

7. Westervelt, R. M. Graphene nanoelectronics. Science 320, 324-325 (2008)

8. Tapasztó, L., Dobrik, G., Lambin, P. \& Biró, L. P. Tailoring the atomic structure of graphene nanoribbons by scanning tunnelling microscope lithography. Nat. Nanotechnol. 3, 397-401 (2008).

9. Geim, A. K. \& Novoselov, K. S. The rise of graphene. Nat Mater 6, 183-191 (2007).

10. Hill, E. W., Geim, A. K., Novoselov, K., Schedin, F. \& Blake, P. Graphene spin valve devices. IEEE Trans. Magn 42, 2694-2696 (2006).

11. Wang, W., Yazyev, O., Meng, S. \& Kaxiras, E. Topological frustration in graphene nanoflakes: magnetic order and spin logic devices. Phys. Rev. Lett. 102, 157201 (2009).

12. Wang, W. L., Meng, S. \& Kaxiras, E. Graphene nanoflakes with large spin. Nano Lett. 8, 241-245 (2007).

13. Pham, B. Q. \& Truong, T. N. Electronic spin transitions in finite-size graphene. Chem. Phys. Lett. 535, 75-79 (2012).

14. Ponomarenko, L. A. et al. Chaotic dirac billiard in graphene quantum dots. Science 320, 356-358 (2008).

15. Lu, J., Yeo, P. S. E., Gan, C. K., Wu, P. \& Loh, K. P. Transforming C60 molecules into graphene quantum dots. Nat. Nanotechnol. 6, 247-252 (2011).

16. Ritter, K. A. \& Lyding, J. W. The influence of edge structure on the electronic properties of graphene quantum dots and nanoribbons. Nat. Mater. 8, 235-242 (2009).

17. Fan, L. \& Zhang, M. Electrochemically generated highly fluorescent graphene quantum dots as a biological label for stem cells. ECS Meeting Abstracts 1201, 1248-1248 (2012).

18. Bonaccorso, F., Sun, Z., Hasan, T. \& Ferrari, A. C. Graphene photonics and optoelectronics. Nat. Photon. 4, 611-622 (2010).

19. Wang, L.-J. et al. A graphene quantum dot with a single electron transistor as an integrated charge sensor. Appl. Phys.Lett. 97, 262113 (2010).

20. Wei, Z. et al. Nanoscale tunable reduction of graphene oxide for graphene electronics. Science 328, 1373-1376 (2010).

21. Hernandez, Y. et al. High-yield production of graphene by liquid-phase exfoliation of graphite. Nat. Nanotechnol. 3, 563-568 (2008).

22. Li, X. et al. Large-area synthesis of high-quality and uniform graphene films on copper foils. Science 324, 1312-1314 (2009).

23. Berger, C. et al. Electronic confinement and coherence in patterned epitaxial graphene. Science 312, 1191-1196 (2006).

24. Wei, D. \& Liu, Y. Controllable synthesis of graphene and its applications. $A d v$. Mater. 22, 3225-3241 (2010).

25. Campos, L. C., Manfrinato, V. R., Sanchez-Yamagishi, J. D., Kong, J. \& JarilloHerrero, P. Anisotropic etching and nanoribbon formation in single-layer graphene. Nano Lett. 9, 2600-2604 (2009).

26. Jiao, L., Zhang, L., Wang, X., Diankov, G. \& Dai, H. Narrow graphene nanoribbons from carbon nanotubes. Nature 458, 877-880 (2009).

27. Pan, D., Zhang, J., Li, Z. \& Wu, M. Hydrothermal route for cutting graphene sheets into blue-luminescent graphene quantum dots. Adv. Mater. 22, 734-738 (2010)

28. Zhuo, S., Shao, M. \& Lee, S.-T. Upconversion and downconversion fluorescent graphene quantum dots: ultrasonic preparation and photocatalysis. ACS Nano 6 , 1059-1064 (2012).

29. Long, D. et al. Fabrication of uniform graphene discs via transversal cutting of carbon nanofibers. ACS Nano 5, 6254-6261 (2011).

30. Son, M., Lim, H., Hong, M. \& Choi, H. C. Direct growth of graphene pad on exfoliated hexagonal boron nitride surface. Nanoscale 3, 3089 (2011).

31. Cong, C. X. et al. Fabrication of graphene nanodisk arrays using nanosphere lithography. J. Phys. Chem. C 113, 6529-6532 (2009).

32. Yazyev, O. V. \& Helm, L. Defect-induced magnetism in graphene. Phy. Rev. B 75, 125408 (2007).

33. Palacios, J. J., Fernández-Rossier, J. \& Brey, L. Vacancy-induced magnetism in graphene and graphene ribbons. Phy. Rev. B 77, 195428 (2008).

34. Lehtinen, P. O., Foster, A. S., Ma, Y., Krasheninnikov, A. V. \& Nieminen, R. M. Irradiation-induced magnetism in graphite: a density functional study. Phy.l Rev. Lett. 93, 187202 (2004).

35. Loh, K. P., Bao, Q., Eda, G. \& Chhowalla, M. Graphene oxide as a chemically tunable platform for optical applications. Nat. Chem. 2, 1015-1024 (2010). 
36. Banhart, F., Kotakoski, J. \& Krasheninnikov, A. V. Structural defects in graphene. ACS Nano 5, 26-41 (2010).

37. Nemanich, R. \& Solin, S. First- and second-order Raman scattering from finitesize crystals of graphite. Phy. Rev. B 20, 392-401 (1979).

38. Graf, D. et al. Spatially resolved raman spectroscopy of single- and few-layer graphene. Nano Lett. 7, 238-242 (2007).

39. McFeely, F. R. et al. X-ray photoemission studies of diamond, graphite, and glassy carbon valence bands. Phy. Rev. B 9, 5268-5278 (1974).

40. Gupta, V. et al. Luminscent graphene quantum dots for organic photovoltaic devices. J. Am. Chem. Soc. 133, 9960-9963 (2011)

41. Zhang, Y., Mori, T., Niu, L. \& Ye, J. Non-covalent doping of graphitic carbon nitride polymer with graphene: controlled electronic structure and enhanced optoelectronic conversion. Energy Environ. Sci. 4, 4517-4521 (2011).

42. Mei, Q. \& Zhang, Z. Photoluminescent graphene oxide ink to print sensors onto microporous membranes for versatile visualization bioassays. Angew. Chem. Int. Ed. 51, 5602-5606 (2012).

43. Mei, Q. et al. Highly efficient photoluminescent graphene oxide with tunable surface properties. Chem. Commun. 46, 7319-7321 (2010).

44. Xin, G. et al. Tunable photoluminescence of graphene oxide from near-ultraviolet to blue. Mater. Lett. 74, 71-73 (2012).

45. Ramón, M. E. et al. CMOS-compatible synthesis of large-area, high-mobility graphene by chemical vapor deposition of acetylene on cobalt thin films. ACS Nano 5, 7198-7204 (2011).

46. Bhaviripudi, S., Jia, X., Dresselhaus, M. S. \& Kong, J. Role of kinetic factors in chemical vapor deposition synthesis of uniform large area graphene using copper catalyst. Nano Lett. 10, 4128-4133 (2010).

47. Ago, H. et al. Epitaxial chemical vapor deposition growth of single-layer graphene over cobalt film crystallized on sapphire. ACS Nano 4, 7407-7414 (2010).

48. Liu, N. et al. Universal segregation growth approach to wafer-size graphene from non-noble metals. Nano Lett. 11, 297-303 (2011).

\section{Acknowledgements}

This work was supported by the Major International (Regional) Joint Research Project of NNSFC (51210002), the link project of the National Natural Science Foundation of China and Guangdong Province (U1034003), the National Natural Science Foundation of China (21073241), the Specialized Research Fund for the Doctoral Program of Higher Education of China (20110171110024) and Australia Research Council (DP120102325 and DP120104932).

\section{Author contributions}

C.Y.H. and P.K.S. conceived the experiments. C.Y.H. performed the experiments. C.Y.H., P.K.S. and S.P.J wrote the paper. All authors contributed through scientific discussions.

\section{Additional information}

Supplementary information accompanies this paper at http://www.nature.com/ scientificreports

Competing financial interests: The authors declare no competing financial interests.

How to cite this article: He, C., Jiang, S.P. \& Shen, P.K. Large-scale and Rapid Synthesis of Disk-Shaped and Nano-Sized Graphene. Sci. Rep. 3, 2144; DOI:10.1038/srep02144 (2013).

(c) (1) (-) $\odot$ This work is licensed under a Creative Commons Attribution-

By No NonCommercial-NoDerivs 3.0 Unported license. To view a copy of this license, visit http://creativecommons.org/licenses/by-nc-nd/3.0 